\title{
Competence in chronic mental illness: the relevance of practical wisdom
}

\author{
Guy A M Widdershoven, ${ }^{1}$ Andrea Ruissen, ${ }^{1}$ Anton J L M van Balkom, ${ }^{2}$ \\ Gerben Meynen ${ }^{3}$
}

'Department of Medical Humanities, VU University Medical Center, Amsterdam, The Netherlands

2Department of Psychiatry, GGZ inGeest, Amsterdam, The Netherlands ${ }^{3}$ Department of Law, Tilburg University, Tilburg, The Netherlands

\section{Correspondence to} Andrea Ruissen, Department of Medical Humanities, VU University Medical Center, Vd Boechorststraat 7, Amsterdam 1081 BL, The Netherlands; ruissen@emergis.nl

Received 14 January 2015 Revised 9 November 2015 Accepted 20 April 2016 Published Online First 10 May 2016

\section{Linked}

- http://dx.doi.org/10.1136/ medethics-2015-103284

- http://dx.doi.org/10.1136/ medethics-2015-103315

- http://dx.doi.org/10.1136/ medethics-2016-103631

CrossMark

To cite: Widdershoven GAM, Ruissen A, van Balkom AJLM, et al. J Med Ethics

2017:43:374-378

\section{ABSTRACT}

The concept of competence is central to healthcare because informed consent can only be obtained from a competent patient. The standard approach to competence focuses on cognitive abilities. Several authors have challenged this approach by emphasising the role of emotions and values. Combining cognition, emotion and values, we suggest an approach which is based on the notion of practical wisdom. This focuses on knowledge and on determining what is important in a specific situation and finding a balance between various values, which are enacted in an individual's personal life. Our approach is illustrated by two cases of patients with obsessive-compulsive disorder.

\section{INTRODUCTION}

The concept of competence is central to healthcare because informed consent can only be obtained from a competent patient. Often, competence is defined in terms of cognitive abilities. Other approaches take emotions and values into consideration. In this paper, we plead for an approach which combines knowledge, emotion and values into the concept of practical wisdom. We illustrate the differences between the approaches by applying them to two obsessive-compulsive disorder (OCD) cases, with chronic courses. This type of disorder differs considerably from 'paradigmatic' incompetence cases of dementia and delusion. In addition, the decisions made in these cases concern longterm treatment commitments rather than choosing or refusing a single intervention, such as an operation. We will argue that these cases show the added value of a practical wisdom approach to competence.

After obtaining informed consent for research we conducted in-depth interviews with a number of OCD patients and their psychiatrists. We asked both the patient and the psychiatrist to tell us about the role of the disease in the patient's life and to reflect on treatment decisions in the (recent) past. We also asked them to evaluate the patient's competence concerning these treatment decisions. Since this evaluation of competence by the patient and the psychiatrist is a reconstruction, it can be biased, but the narrative structure of the interviews provides relevant contextual information since treatment decisions are regarded as elements of the story in which patient and psychiatrist make sense of crucial events in the patient's life. We also assessed competence using the MacCAT retrospectively. ${ }^{1}$ This gives an indication of the outcome of a MacCAT-assisted assessment; however, it has limitations, since the application is post hoc and is not combined with a psychiatric assessment. Yet, such an approach has been used before in a study on competence in psychiatry. ${ }^{1}$

In this paper, we focus on anonymised data from the interviews with two patients, which we call Jack and Mary, and their psychiatrists. Our interpretation of the two cases is supported by the stories of other patients and their psychiatrists.

\section{CASES}

Jack, 55, has suffered from OCD for 20 years. The symptoms started quite acute. After his divorce, he found himself avoiding streets with garbage bags. He knew that there was no rationale for this, but he kept on doing it. He washed himself more often, to be sure that he was not dirty. In about half a year, the symptoms increased so much that he could not work anymore, slept really bad and consumed more and more alcohol. He did not answer the telephone, ate poorly and spent hours showering, washing and cleaning according to a specific ritual. About his symptoms he says: "it is idiotic, one cannot understand oneself, and I still cannot understand myself after twenty years."

He was able to hide his symptoms, and his children, who were young at that time, did not notice much. Yet, his sister and brother became worried and visited his house. At first, he did not allow them to enter the house. When he finally let them in, they were shocked: a house full of dirt, a mess and soap scums, Jack mainly lying on his bed and doing nothing. They brought the family physician to his house.

The doctor saw a cachectic man, in a chaotic environment and without social contacts. Yet, he was verbally strong and clearly stated that he did not want any help. When confronted with his extreme behaviour, he acknowledged that he was different from other people, but that he preferred living his life in his own way. Against Jack's wish, an admission to a psychiatric hospital was arranged. It is unknown whether the psychiatrist deemed Jack incompetent, because the key criterion for compulsory confinement in Dutch psychiatry law is danger, not incompetence. Looking back, Jack praises the doctor and says "I was lost in strange behaviour."

After admission, he was treated and his symptoms became less severe. He was discharged, but at home his situation did not improve very much.

\footnotetext{
${ }^{\mathrm{i}}$ Anonymisation means that neither the patient nor anyone else could identify the patient. Detail has been removed from these case descriptions to ensure anonymity.
} 
Over the years, he had various treatments, including medication and cognitive behavioural therapy. The results were minimal. About 5 years ago, Jack visited a new psychiatrist and heard about Deep Brain Stimulation (DBS). The psychiatrist explained that he should first try all other options. After a period ofagain-medication and psychotherapy, Jack was treated with DBS. At first, nothing changed, but after about a year, some results showed. His symptoms are in remission now, but not gone. $\mathrm{He}$ is still occupied with compulsive behaviour several hours a day. He regularly visits the psychiatrist. He aims to develop social contacts, and considers moving to another house. His relationship with his family has improved, and he has time to work on other symptoms, like lack of sleep. Jack says about his current situation: "I lived on another planet for about twenty years, it seems. I now am able to cope with other people, and go to the supermarket." Although Jack is doing better, he does not have a job and has no intimate relations; yet, he considers discontinuing treatment.

Jack thinks he was incompetent at the time of admission, and his current psychiatrist, knowing his history and his current way of dealing with his situation, agrees. Based on the MacCAT criteria, applied retrospectively, he was competent to refuse admission, as he recognised that his behaviour was peculiar and gave reasons for his refusal of treatment. Jack now feels competent. His psychiatrist is in doubt, as he thinks that Jack is too optimistic about his achievements and does not acknowledge that he needs support to further improve his living condition. Yet, the psychiatrist does not see strict indications for incompetence. Based on the MacCAT criteria, Jack is competent to refuse continuation of treatment. For MacCAT scores, see table 1.

Mary, age 60, was diagnosed with OCD nearly 40 years ago. Her symptoms include the urge to shower and wash with particular rituals, and housekeeping following a rigid protocol: "I have to do it the way it has to be done." If she does not follow the rituals or the protocol, she gets nervous. The same happens when her schedule is disturbed, for example, when the girlfriend of her son comes by unexpectedly.

She is very proud of her son, who is now 20 years old. She had difficulties in becoming pregnant; it took 11 years. Due to her symptoms, she could not take care of the baby properly. Her husband took over the care: washing and changing diapers. She still feels guilty about that. Five years later, she became pregnant again, but this pregnancy had to be ended because of kidney failure of the fetus. This still haunts her. Being a mother is important for her, and having only one child keeps disturbing her.

Mary was admitted several times and treated with antidepressants and cognitive behavioural therapy. She experienced these interventions as burdensome and tells that she felt 'fed up', because they did not help very much. She decided to stop treatment. Looking back at this decision, she says she had no reasons, but just could not set herself to continuing. Reflecting on her decision to stop, she says that she experienced therapy as stressful and wanted to avoid trying new things, which interfered too much with her established way of living.

Three years ago, she started seeing a psychiatrist. He proposed daytime activities, like babysitting or lunch assistance at a primary school, since she was interested in caring for children. Mary agreed, not regarding this as therapy, but as an advice from a friend. Mary is doing better now: she still has obsessivecompulsive symptoms, including cognitive problems such as loss of concentration and problems with memory, but she is able to live her life in a satisfactory way and is a popular babysitter.

Mary thinks that she was competent when she ended her treatments in the past and is so now. Her current psychiatrist

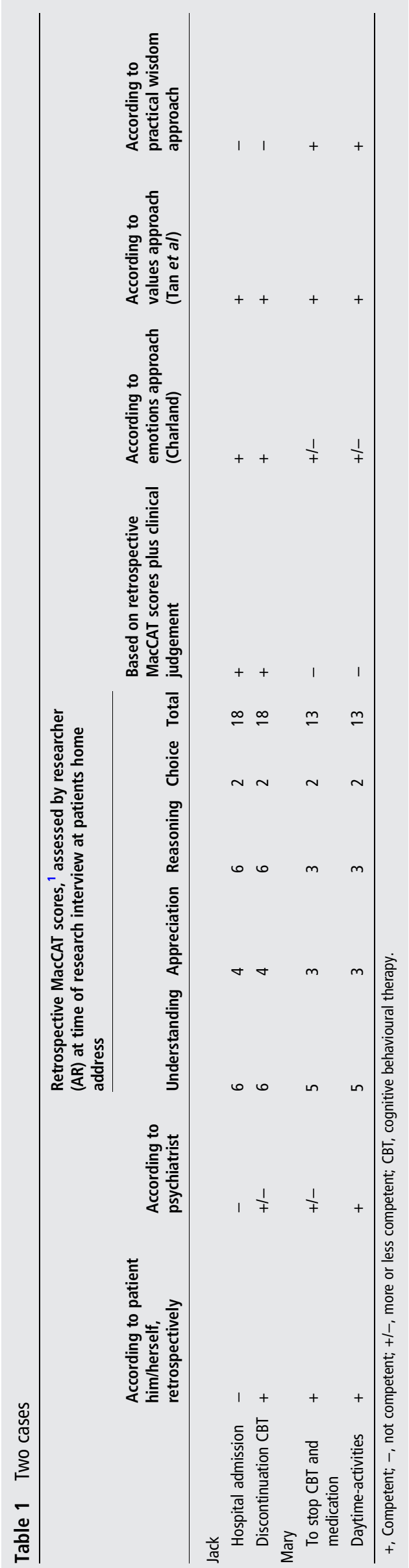


doubts whether she was competent when stopping treatment, since she did not seem to understand that therapy necessarily includes changing one's way of life. He regards her decision to try baby-sitting as competent, since she shows some understanding of its beneficial effects. He regards it as positive that she has proven capable of integrating a significant life event (loss of a baby) in her rehabilitation attempts. Based on the MacCAT criteria, she was neither competent to refuse further treatment, since she did not understand the preconditions and effects of therapy, nor to consent to daytime activities, since she lacked insight into its therapeutic aims. For MacCAT scores, see table 1.

\section{DIFFERENT APPROACHES TO COMPETENCE A cognitive approach}

A commonly used approach to competence focuses on the ability to express a choice, to reason about treatment options, to appreciate a situation and its consequences and to understand relevant information. These are also the key elements of the MacCAT. The MacCAT should be combined with clinical judgement to make a final decision about a patients' competence. ${ }^{2}$ This approach to competence emphasises cognitive abilities, such as understanding information and reasoning. The element of appreciation, implying that the patient recognises the existence of a disorder and the likely impact of the treatment choices on his or her life, also implies cognitive judgement.

When applied to the case of Jack, the cognitive approach seems to lead to the conclusion that he was competent when he refused admission in the past. He showed awareness that his behaviour was peculiar and was able to argue that his rituals were meaningful to him. His current cognitive abilities also indicate competence, since he is able to understand information about therapy and to apply it to his situation. The outcome of his reasoning is that he does not regard further therapy necessary, as he is content with his improvements so far. This is not in line with the view of the psychiatrist, but that in itself does not make Jack incompetent.

From a cognitive perspective, Mary does not seem to be competent in her decision to stop treatment and later to accept day-time activities, since she does not provide any reasons and did not seem to balance the pros and cons of both decisions in a neat way. Her decision to stop treatment is only explained by reference to a feeling of 'being fed up'. Her acceptance of the proposal for day activities is not based on appreciation of the situation, as it does not show awareness of the nature of the illness and the role of therapy.

Against the cognitive approach, it has been argued that cognitive abilities are neither necessary nor sufficient for adequate decision making. ${ }^{3} 4$ Some patients may have logical, valid arguments for accepting or refusing treatment but may show little insight into the illness and its consequences for their life. ${ }^{1} 34$ Other patients may lack the ability to argue logically, but the decision may show congruence with the patient's way of living and life goals. This can be seen in the cases of Jack and Mary. In hindsight, Jack admits that his refusal of being admitted was not properly motivated. His current consideration to stop treatment may also be doubted, since he is unable to organise his life in a meaningful way. Mary's decisions, on the other hand, seem to indicate that she knows what is important for her and is able to manage. Her decision to stop treatment was not based on reasoning, but motivated by the stress she experienced, which she somehow seemed to interpret as a risk to her well-being. When she accepted the psychiatrist's proposal to try baby-sitting, this fitted in with her interest in caring for children.

\section{Emotions}

Charland ${ }^{5}$ suggests taking emotions explicitly into account in judgements of competence. In his paper, Is Mr. Spock mentally competent?, he argues that competence 'requires the positive contribution of emotion, something Spock is incapable of'. Consequently, he pleas for a modification of the existing tests and models to make them 'appropriate to the kinds of practical decision-making situations they are ostensibly designed for'.

If we apply this approach to the case of Jack, we can see that his refusal to being admitted and his inclination to stop cognitive behavioural therapy do not lack emotions. He is emotionally involved in these decisions, stressing that he wants to be in control over his life. Next to this fundamental emotion, Jack has a range of other emotions, such as love for his children and the need to be loved. The problem is that he is unable to translate these into decisions and actions, which result in a balanced life in relation with others. This, however, is not enough to make him incompetent in Charland's conception.

Mary's decision to end treatment showed emotion, as she was 'fed up'. In general, 'being fed up' is regarded as a negative emotion, leading towards a biased decision. However, in line with Charland's approach, one might say that in this case the emotion was positive in that it expressed the value of leading a peaceful life, and avoiding stress, which for Mary was evidently important. Thus, the emotion seems to have made a positive contribution to the decision. Mary's decision to accept the physician's proposal concerning day care is related to a clearly positive emotion, which is love for children. It shows Mary trusts that the decision is well founded, although she cannot give explicit reasons for it.

In summary, an approach in terms of emotions does not by itself lead to questioning Jack's competence. As his decisions clearly show emotional involvement, the problem is not a lack of emotions, but a balance between the emotions which accompany the decision and other emotions which are relevant to him. An approach in terms of emotions does provide indications that Mary is competent, since her decisions seem to be accompanied by proper emotions and can thus be regarded as justified, although adequate reasoning is absent.

\section{Values}

A further challenge to the traditional view of competence is provided by Tan et al. ${ }^{167}$ The authors interviewed female patients diagnosed with anorexia nervosa, aiming to identify aspects of thinking that might be relevant to the issue of competence to refuse treatment'. Anorexia nervosa, they suggest, can compromise patient decision-making by fundamentally changing a patient's values, like 'being slim is more important than being happy'. Tan et al consider anorexia-related values to be 'pathological'. They emphasise that, in retrospect, these anorexic patients 'performed very well on the MacCAT-T'. They were able to understand the risks involved in not eating and to reason logically. However, anorexia nervosa compromises competence, in that the patients base their decisions on values which they would not endorse without their illness. This implies an approach to competence in which pathology is central. ${ }^{89}$

If we apply this approach to the case of Jack, we see that he is very much focused on the value of 'cleanliness'. His urge to constantly wash his hands and other items is clearly motivated by the importance he attaches to being clean, and he structures his life around this core value. Yet, neither his disagreement with admission nor his inclination to stop cognitive behavioural treatment originates in an overemphasis of the value of 
cleanliness, compared with other values. He does not refuse treatment because he deems being clean more important than what could be considered leading a meaningful life, but simply does not see what he needs for the latter.

In the case of Mary, the decision to stop treatment was not motivated by the urge to be clean, but by her experience that treatment did not help her to build a more meaningful life. Her later decision to follow the physician's advice to take up daytime activities involving caring for children can be said to be in line with a pre-existing, non-pathological value, caring for children. Therefore, following Tan's line of argument, one might argue that this decision was competent.

In summary, the value approach in Jack's case does not seem to lead to other conclusions than the cognitive approach. In the case of Mary, it does provide indications for revising the assessment of competence on the basis of her values, not by comparing pathological values with normal ones, but by shedding light on the importance of being able to organise one's life in accordance with fundamental values. In both cases, the ability to implement one's decision in life is lacking.

\section{Practical wisdom}

We propose an alternative approach to competence, following Aristotle's notion of practical wisdom (phronesis). ${ }^{10}$ First, practical wisdom implies knowledge of what fits into the situation and what is the right thing to do. ${ }^{11}$ Practical wisdom entails being able to apply general rules to the concrete situation. This is not just a matter of intuition, as it requires knowledge of the situation, which can be made explicit and justified afterwards. Second, practical wisdom is emotional; ${ }^{12}$ it implies having an adequate disposition and having adequate feelings about right and wrong. Practical wisdom means being able to find a balance between extreme feelings, for instance, being too involved with others on the one hand and showing no interest in their wellbeing on the other hand. Third, practical wisdom enables a person to combine different values in life, such as work and leisure, relationships and independence, and activity and rest. The right balance cannot be established in a theoretical way but needs to be enacted in practice, taking into account and accepting one's capacities as well as limitations. ${ }^{13}$

In terms of practical wisdom, Jack can be regarded as incompetent, both in the past and in the present. At the time of admission, he lacked the ability to deal with his situation and did not know how to live with his symptoms. His intention to end cognitive behavioural therapy shows a lack of insight into the need of support in finding ways of dealing with his limitations. From the perspective of practical wisdom, Mary, on the other hand, shows competence. Her earlier decision to end treatment can be seen as the expression of insight that it did not help her. Her recent choice to try new daily activities fits in with her values and enables her to find a new balance in life.

The approach in terms of practical wisdom supports the view of both Jack and his psychiatrist that his refusal to being admitted was unwise. It is also congruent with both Mary's and her psychiatrist's view on her acceptance of the proposal for daily activities. The practical wisdom approach is better able to support these shared judgements than the other approaches to competence. Yet, the perspective of practical wisdom can also lead to conclusions, which differ from that of the patient or the psychiatrist. First, it is not in line with Jack's view that he is currently competent to decide about stopping further treatment. Second, it is not congruent with the view of Mary's psychiatrist that she seems to have been incompetent when stopping treatment in the past. In both cases, there is a discrepancy between the judgement of the patient and the psychiatrist. The practical wisdom approach in these cases does not automatically support the view of the patient or that of the psychiatrist. It does question the reliance on (lack of) cognitive reasoning as the decisive criterion for (in)competence.

\section{CONCLUSION}

The concept of practical wisdom combines knowledge, emotions and values. With the cognitive approach, it shares the view that competence requires knowledge, although this need not be explicit and argumentative. In line with Charland and Tan, it takes into consideration that competence is personal, embodied and value-laden. The notion of practical wisdom focuses on (1) knowing the right thing to do in the concrete situation, (2) having adequate emotions and (3) being able to find a balance between various values, and enact them in personal life. The notion of practical wisdom does not necessarily involve a higher threshold for competence, since a person, to some extent, may lack the capacity of cognitive reasoning but may nevertheless have practical insight and be able to handle the situation and live a meaningful life.

Applied to the cases of Jack and Mary, the concept of practical wisdom leads to outcomes of competence assessments, which are different from approaches focusing on either cognition, or emotions, or values. From the perspective of practical wisdom, Jack is incompetent, both when refusing being admitted to the hospital and when considering stopping treatment, although he is able to give reasons. On the other hand, Mary is competent, because she shows practical understanding, both when stopping treatment and when agreeing to try baby-sitting, although she lacks the capacity to explain her decisions through reasoning and argumentation. These conclusions are more in line with the retrospective judgements about competence of the patients and their psychiatrists than the outcomes of other approaches to competence.

We conclude that the notion of competence as practical wisdom encompasses relevant notions addressed in other approaches and can provide psychiatrists with criteria to decide on whether or not to respect a patient's wishes, which do justice to the complexity of care for psychiatric patients suffering from OCD. Further research should clarify whether this approach is also useful for other chronic psychiatric conditions and has relevance for more acute psychiatric illnesses too.

Contributors GAMW, AR, AJLMvB and GM contributed to the conception and design of the work, and analysis and interpretation of data for the work, gave final approval of the version to be published and agree to be accountable for all aspects of the work in ensuring that questions related to the accuracy or integrity of any part of the work are appropriately investigated and resolved. GAMW, AR and AJLMvB contributed to the acquisition. GAMW and AR drafted the work. AJLMvB and GM revised the work critically for important intellectual content.

Competing interests None declared.

Ethics approval Ethics Committee VU Medical Centre, Amsterdam.

Provenance and peer review Not commissioned; externally peer reviewed.

\section{REFERENCES}

1 Tan DJ, Hope PT, Stewart DA, et al. Competence to make treatment decisions in anorexia nervosa: thinking processes and values. Philos Psychiat Psychol 2006;13:267-82

2 Grisso T, Appelbaum PS, Hill-Fotouhi C. The MacCAT-T: a clinical tool to assess patients' capacities to make treatment decisions. Psychiatr Serv 1997;48:1415-19.

3 Ruissen A, Meynen G, Widdershoven GAM. Perspectives on patient competence in psychiatry: cognitive functions, emotions and values. Tijdschr Psychiatr 2011;53:405-14 


\section{Viewpoint}

4 Ruissen AM, Widdershoven GA, Meynen G, et al. A systematic review of the literature about competence and poor insight. Acta Psychiatr Scand 2012;125:103-13.

5 Charland LC. Is Mr. Spock Mentally Competent? Competence to Consent and Emotion. Philos Psychiat Psychol 1998;5:67-81.

6 Tan J. The anorexia talking? Lancet 2003:362:1246.

7 Tan J. Bridging the gap between fact and values. World Psychiatry 2005:4:92-3.

8 Meynen G, Widdershoven GAM. Emotionality and competence: changing emotions versus dealing with emotions. Am J Bioeth Neurosci 2011;2:64-6.
9 Meynen G, Widdershoven GAM. Competence in health care: an abilities-based versus a pathology-based approach. Clin Ethics 2012;7:39-44.

10 Aristotle. The nicomachean ethics. Cambridge, Massachusetts: Harvard University Press, 1975.

11 Sorabji R. Aristotle on the Role of Intellect in Virtue. In: Rorty AO, ed. Essays on Aristotle's ethics. Berkeley: University of California Press, 1980.

12 Nussbaum MC. Upheavals of thought. The intelligence of the emotions. Cambridge: Cambridge University Press, 2001.

13 Nussbaum MC. The Fragility of goodness. Luck and ethics in Greek tragedy and philosophy. Cambridge: Cambridge University Press, 1986. 